\title{
Quantum production of black holes at colliders
}

\author{
Nicusor Arsene ${ }^{1,2, a}$, Roberto Casadio ${ }^{3,4, b}$, Octavian Micu $^{1, c}$ \\ ${ }^{1}$ Institute of Space Science, Bucharest, P.O. Box MG-23, 077125 Bucharest-Magurele, Romania \\ ${ }^{2}$ Physics Department, University of Bucharest, Bucharest-Magurele, Romania \\ ${ }^{3}$ Dipartimento di Fisica e Astronomia, Alma Mater Università di Bologna, via Irnerio 46, 40126 Bologna, Italy \\ ${ }^{4}$ I.N.F.N., Sezione di Bologna, viale Berti Pichat 6/2, 40127 Bologna, Italy
}

Received: 22 February 2016 / Accepted: 23 June 2016 / Published online: 8 July 2016

(c) The Author(s) 2016. This article is published with open access at Springerlink.com

\begin{abstract}
We investigate black hole production in $p p$ collisions at the Large Hadron Collider by employing the horizon quantum mechanics for models of gravity with extra spatial dimensions. This approach can be applied to processes around the fundamental gravitational scale and naturally yields a suppression below the fundamental gravitational scale and for increasing number of extra dimensions. The results of numerical simulations performed with the black hole event generator BLACKMAX are here reported in order to illustrate the main differences in the numbers of expected black hole events and mass distributions.
\end{abstract}

\section{Introduction}

The possibility to produce black holes (BHs) at particle colliders is directly related to the question whether the fundamental gravitational scale is somewhere in the few $\mathrm{TeV}$ range, as was suggested in scenarios with extra spatial dimension, like the ADD model [1,2] and the RS model [3,4] (see also Refs. [5,6] for a comprehensive review). Above the gravitational scale, it is generally expected that BHs can be created and finding signatures of their decays would be evidence in favour of these extra-dimensional models [5,7]. During the recent years, it was proposed that high energy particle colliders could turn out to be huge $\mathrm{BH}$ factories $[8,9]$, and there have actually been many searches to observe the production and decay of semiclassical and quantum BHs at

\footnotetext{
a e-mail: nicusorarsene@spacescience.ro

be-mail: casadio@bo.infn.it

c e-mail: octavian.micu@spacescience.ro
}

the LHC. ${ }^{1}$ The ATLAS collaboration looked for events with jet+leptons $[12,13]$ or dimuon [14] in the final state of $p p$ collisions at a centre-of-mass energy of $\sqrt{s}=8 \mathrm{TeV}$. At the same time, the CMS collaboration was searching for energetic multi-particle final states, as well as for resonances and quantum black holes using the dijet mass spectra at $\sqrt{s}=8 \mathrm{TeV}[15,16]$. These searches and their results are very important for the community, especially in the context of the existing extra-dimensional models. They represent direct comparisons between an experiment and the theoretical predictions for new physics at these energies and can be used to constrain the parameters of the models [17]. For example, the CMS collaboration [15] excluded the production of quantum/semiclassical BHs with masses below 4.3 to $6.2 \mathrm{TeV}$ (depending on the models) with $95 \%$ confidence level, while ATLAS results indicate the threshold mass of the quantum $\mathrm{BH}$ to be larger than $5.3 \mathrm{TeV}$ [13]. However, this exclusion limits are strongly dependent on the $\mathrm{BH}$ production cross section and different decay modes.

Here, we analyse the $\mathrm{BH}$ production by employing the modified cross section in the ADD model [1,2] obtained in Ref. [18] from the Horizon Quantum Mechanics (HQM) of localised sources [19-25]. In fact, this approach was specifically devised to yield the probability that a particle is a $\mathrm{BH}$, and is therefore perfectly suited to address this issue. To perform the analysis, we then adapt the BLACKMAX code [26], one of the most powerful and widely used BH event generators, which includes different scenarios like tension/tensionless rotating/non-rotating BHs. ${ }^{2}$ The results of our findings will be presented in Sect. 3. Before that, we familiarise the reader with the HQM and provide some use-

\footnotetext{
1 A BH is considered semiclassical if it decays via Hawking radiation, whereas it is generically called quantum if the decay is not thermal, including the case of a stable remnant $[10,11]$.

2 Examples of other available $\mathrm{BH}$ event generators are CHARYBDIS2 [27], QBH [28] and CATFISH [29].
} 
ful references for a more in-depth study of the formalism in Sect. 2.

\section{Horizon quantum mechanics}

The HQM for static sources [19-24] was extended to higher dimensions in Refs. [18,25], which can be naturally applied to BHs in the ADD scenario. Let us start by considering the wave-function for a localised massive particle as given by a spherically symmetric Gaussian wave-packet of width $\ell$ in $D$ spatial dimensions [representing a source in a $(D+1)$ dimensional space-time],

$\psi_{\mathrm{S}}(r)=\frac{e^{-\frac{r^{2}}{2 \ell^{2}}}}{(\ell \sqrt{\pi})^{D / 2}}$,

whose form in momentum space is

$\tilde{\psi}_{\mathrm{S}}(p)=\frac{e^{-\frac{p^{2}}{2 \Delta^{2}}}}{(\Delta \sqrt{\pi})^{D / 2}}$,

where $\Delta=\hbar / \ell=m_{D} \ell_{D} / \ell, m_{D}$ is the fundamental gravitational mass and $\ell_{D}=\hbar / m_{D}$ represents the corresponding length scale. We study the simplest case and assume that when a BH forms, it will be described by the $(D+1)$ dimensional Schwarzschild metric,

$$
\begin{aligned}
\mathrm{d} s^{2}= & -\left(1-\frac{R_{D}}{r^{D-2}}\right) \mathrm{d} t^{2}+\left(1-\frac{R_{D}}{r^{D-2}}\right)^{-1} \mathrm{~d} r^{2} \\
& +r^{D-1} \mathrm{~d} \Omega_{D-1},
\end{aligned}
$$

where the classical horizon radius is given by

$R_{D}(M)=\left(\frac{2 G_{D} M}{|D-2|}\right)^{\frac{1}{D-2}}$,

and $G_{D}=\ell_{D}^{D-2} / m_{D}$ represents the fundamental gravitational constant in this ADD scenario.

We now consider the mass-shell relation in flat space, $p^{2}=E^{2}-m^{2}$, where $m$ is the rest mass of the source, and express $E$ in terms of the above horizon radius (4), $r_{\mathrm{H}}=R_{D}(E)$. After using these results in Eq. (2), the normalised horizon wave-function reads [18]

$\psi_{\mathrm{H}}\left(r_{\mathrm{H}}\right)=\mathcal{N} \Theta\left(r_{\mathrm{H}}-R_{D}(m)\right) e^{-\frac{(D-2)^{2} m_{D}^{2}}{8 \Delta^{2}}\left(\frac{r_{\mathrm{H}}}{\ell_{D}}\right)^{2(D-2)}}$,

where the normalisation $\mathcal{N}$ is obtained from the Schrödinger scalar product in $D$ spatial dimensions and the step function ensures that the gravitational radius $r_{\mathrm{H}} \geq R_{D}(m)$, since $m$ is the minimum energy eigenvalue contributing to the packet. We can now calculate the probability for the particle to be a $\mathrm{BH}$,

$P_{\mathrm{BH}}=\int_{0}^{\infty} \mathcal{P}_{<}\left(r<r_{\mathrm{H}}\right) \mathrm{d} r_{\mathrm{H}}$

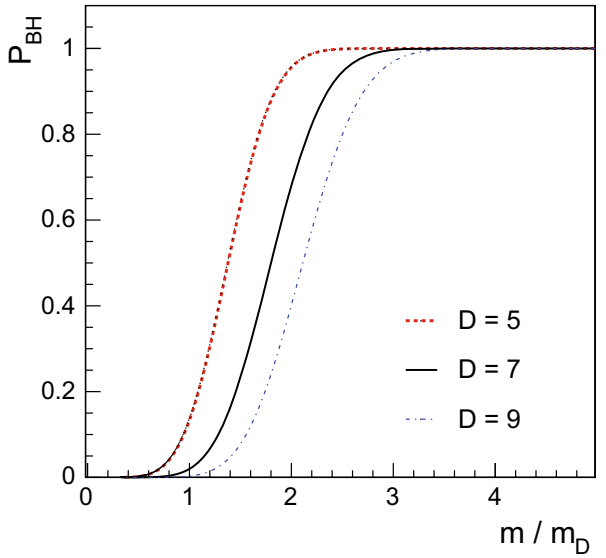

Fig. 1 Probability (7) for a particle described by a Gaussian wavefunction of mass $m$ to be a BH for different numbers of spatial dimensions $D$

where $\mathcal{P}_{<}\left(r<r_{\mathrm{H}}\right)$ represents the probability density for the particle to be inside its own gravitational radius $r_{\mathrm{H}}$ and is the product of two factors: the probability for the particle to be located inside a $D$-ball of radius $r_{\mathrm{H}}$ and the probability density that the horizon radius equals $r_{\mathrm{H}}$. In this particular case, the BH probability depends on the Gaussian width $\ell$, particle mass $m$ and number of spatial dimensions $D$. We can further assume $\ell=\lambda_{m}=m_{D} \ell_{D} / m$ is the Compton length of the source, which represents the minimum uncertainty in its size, so that $\Delta=m$ and the probability only depends on $m$ and the number of dimensions $D$ [18],

$$
\begin{aligned}
& P_{\mathrm{BH}}(m ; D)=\left(\frac{D-2}{2}\right)^{\frac{2}{D-2}} \frac{\left(m / m_{D}\right)^{\frac{D}{D-2}}}{\Gamma\left(\frac{D / 2}{D-2}, 1\right) \Gamma\left(\frac{D}{2}\right)} \\
& \quad \times \int_{R_{D}(m)}^{\infty} \gamma\left(\frac{D}{2}, \frac{m^{2} r_{\mathrm{H}}^{2}}{m_{D}^{2} \ell_{D}^{2}}\right) e^{-\left[\frac{(D-2) m_{D}}{2 m}\right]^{2}\left(\frac{r_{\mathrm{H}}}{\ell_{D}}\right)^{2(D-2)}} \frac{r_{\mathrm{H}}^{D-1}}{\ell_{D}^{D}} \mathrm{~d} r_{\mathrm{H}},
\end{aligned}
$$

where $\Gamma(a, b)$ is the upper incomplete gamma function and $\gamma(a, b)$ the lower incomplete gamma function. The above expression can be computed numerically and is displayed in Fig. 1 for $D=5,7$ and 9 .

There are a few important observations regarding this result. First of all, like in $D=3$, there is no sharp threshold for $\mathrm{BH}$ formation, but the $\mathrm{BH}$ probability drops very fast for $m<m_{D}$ (or, equivalently, $\ell>\ell_{D}$ ). Moreover, for any given mass, say $m \simeq m_{D}$, the probability $P_{\mathrm{BH}}(m ; D)$ decreases for increasing values of $D$. In the next section, we will focus on expressing these differences in a more quantitative way.

\section{Cross section $p p \rightarrow \mathrm{BH}$}

We will now focus on the implications for $\mathrm{BH}$ searches at the LHC. As stated in the Introduction, we performed the numer- 

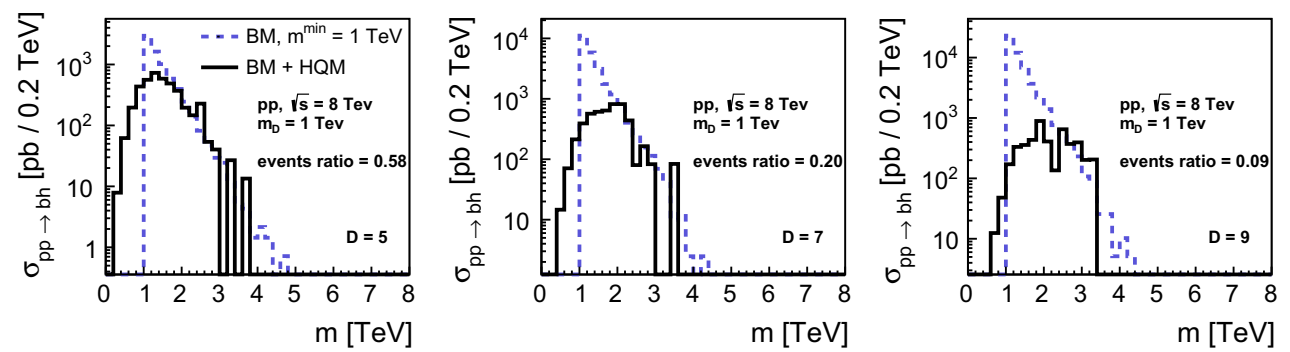

Fig. 2 BH mass distribution, weighted by the cross section for $p$ collision at $\sqrt{s}=8 \mathrm{TeV}$, for a fundamental gravitational mass $m_{D}=1 \mathrm{TeV}$ and number of spatial dimensions $D=5,7$ and 9. Blue dashed lines are for the standard scenario with cross section (8) and $m^{\text {min }}=m_{D}$; contin-
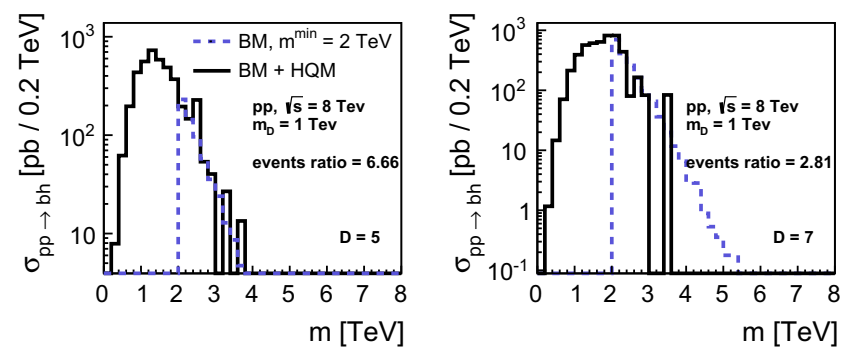

uous black lines represent the modified case (9) and events ratio gives the corresponding suppression factor. Each curve is an average over $10^{4}$ simulations of tensionless non-rotating BHs in BLACKMAX 2.02.0
Fig. $3 \mathrm{BH}$ mass distribution, weighted by the cross section for $p$ collision at $\sqrt{s}=8 \mathrm{TeV}$, for a fundamental gravitational mass $m_{D}=1 \mathrm{TeV}$ and number of spatial dimensions $D=5,7$ and 9. Blue dashed lines are for the standard scenario with cross section (8) and $m^{\min }=2 m_{D}$;

ical simulations using BLACKMAX 2.02.0 and considering tensionless non-rotating BHs. In the standard configuration, BLACKMAX employs the BH production cross section

$\sigma_{\mathrm{BH}}(E)=b_{D}^{2} \pi R_{D}^{2}(E)$,

where $b_{D}=2\left[1+(D-1)^{2} / 4\right]^{\frac{1}{2-D}}$ and $R_{D}$ is the horizon radius (4). Among other parameters, one can set the values of the fundamental gravitational scale $m_{D}$ and the minimum BH mass $m^{\text {min }}$. In fact, it is important to remark that no threshold of $\mathrm{BH}$ production is fixed in the standard scenarios, although one expects that BHs do not form below a certain mass because of quantum fluctuations, and one can at best constrain $m^{\text {min }}$ from the data. Typically, we shall consider $m^{\text {min }} \gtrsim m_{D}$ in order to ensure that no $\mathrm{BH}$ is produced with a mass below $m_{D}$ in the standard case.

In the HQM picture of Refs. [18,25], the effective BH production cross section is instead given by

$\sigma_{\mathrm{HQM}}(E)=P_{\mathrm{BH}}(E) \sigma_{\mathrm{BH}}(E)$,

where $P_{\mathrm{BH}}(E)=P_{\mathrm{BH}}(m=E ; D)$ is the probability (7) for a particle with energy $E$ to be a $\mathrm{BH}$, while $\sigma_{\mathrm{BH}}$ is still given by Eq. (8). Note that there is now no need for imposing a minimum $\mathrm{BH}$ mass, since $P_{\mathrm{BH}}$ acts as a proper quantum regulator. In order to implement this improved cross section, we considered the fundamental gravitational mass scale to have the same value as in the standard case, and set a continuous black lines represent the modified case (9) and the events ratio gives the corresponding suppression factor. Each curve is an average over $10^{4}$ simulations of tensionless non-rotating BHs in BLACKMAX 2.02.0

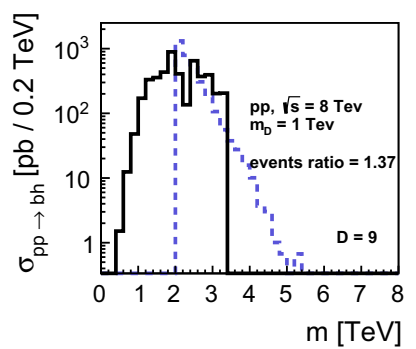

minimum BH mass $m^{\min }=0.2 m_{D}$ for computational convenience. ${ }^{3}$ Finally, we added a subroutine to BLACKMAX which weighs the standard $\mathrm{BH}$ mass distributions with the probability $P_{\mathrm{BH}}(E)$.

We first illustrate the typical differences between the simulations that employ the standard cross section (8) and the HQM cross section (9) in Figs. 2 and 3. Later on, we will investigate more general cases and include comparisons to the current bounds on $m_{D}$ and $m^{\min }$ by the ATLAS and CMS collaborations. The blue dashed lines are obtained by employing the standard $\mathrm{BH}$ production cross section (8) for $p p$ collisions at $\sqrt{s}=8 \mathrm{TeV}$, with $m_{D}=1 \mathrm{TeV}$, and setting the minimum BH mass to $m^{\min }=m_{D}$ (in Fig. 2) or $m^{\text {min }}=2 m_{D}$ (in Fig. 3). The continuous black lines in the same plots represent the analogous BH mass distribution derived from the modified production cross section (9). First of all, in agreement with Fig. 1 and the HQM approach [18,21-23], BHs with masses below the fundamental scale of gravity are now possible and no sharp threshold effect like the one forced in the standard case exists. For $m^{\min }=m_{D}=1 \mathrm{TeV}$, the HQM cross section (9) leads to a significant suppression of $\mathrm{BH}$ production, whereas for $m^{\min }=2 m_{D}=2 \mathrm{TeV}$ the situation is reversed. Besides investigating how the differential production cross section

\footnotetext{
3 We checked the final results do not change significantly when lowering $m^{\mathrm{min}}$ even further.
} 

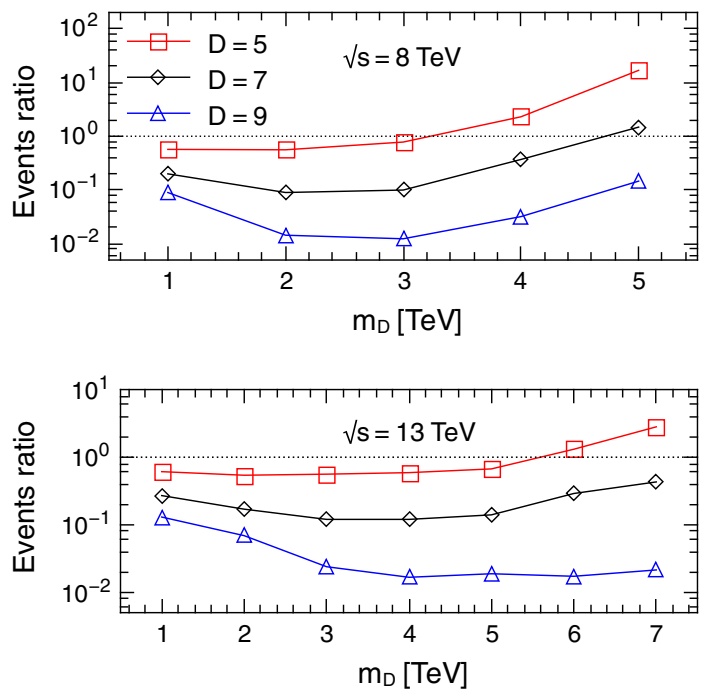

Fig. 4 Dependence of the events ratio on the value of $m_{D}=m^{\text {min }}$ for $\sqrt{s}=8 \mathrm{TeV}$ (top panel) and $\sqrt{s}=13 \mathrm{TeV}$ (bottom panel). Note the logarithmic scale on the vertical axis

varies with the value of the resulting $\mathrm{BH}$ mass, we can also compare the total cross sections in the two cases. This comparison is given by the events ratio, the ratio between the HQM total production cross section relative to the standard case, which we also display in the plots. In particular, the event ratio is smaller than one (thus signalling a suppression) for $m^{\min }=m_{D}=1 \mathrm{TeV}$, but larger than one (indicating an enhancement) for $m^{\min }=2 m_{D}=2 \mathrm{TeV}$. We also notice that this ratio is always smaller for larger $D$, again in agreement with Fig. 1. This can be viewed as a check which makes us confident that our numerical simulations are accurate.

In light of these preliminary results, it appeared interesting to study how the events ratio depends on the value of the fundamental gravitational scale for different numbers of spatial dimensions. This analysis is presented in Fig. 4 for $m_{D}=m^{\mathrm{min}}$. We see that, in this case, regardless of the value of $m_{D}$, this ratio is always smaller for larger number of extra-dimensions (at the same value of the gravity scale). Another feature we notice is that, for all numbers of spatial dimensions, from around $m_{D}=2 \mathrm{TeV}$ for $\sqrt{s}=8 \mathrm{TeV}$ (respectively $m_{D}=4 \mathrm{TeV}$ for $\sqrt{s}=13 \mathrm{TeV}$ ) the events ratio starts to increase with $m_{D}$, eventually crossing unity from below. Even though it seems that more BHs are produced in the HQM scenario than in the standard case for higher values of $m_{D}$, we have to remember that the total cross section decreases with $D$ and the number of expected BH events remains very small. This dumping of the total cross sections for increasing $m_{D}$ is exemplified in Fig. 5 for $D=6$ (but the same behaviour holds in all cases): for smaller values of $m_{D}$, the standard production is larger than the HQM expectation, and the two cross at a relatively large value of $m_{D}$ (which depends on $D$ ). As one can see, even where $\sigma_{p+p \rightarrow \text { BH }}$ predicted by the HQM is larger, the actual values of the total
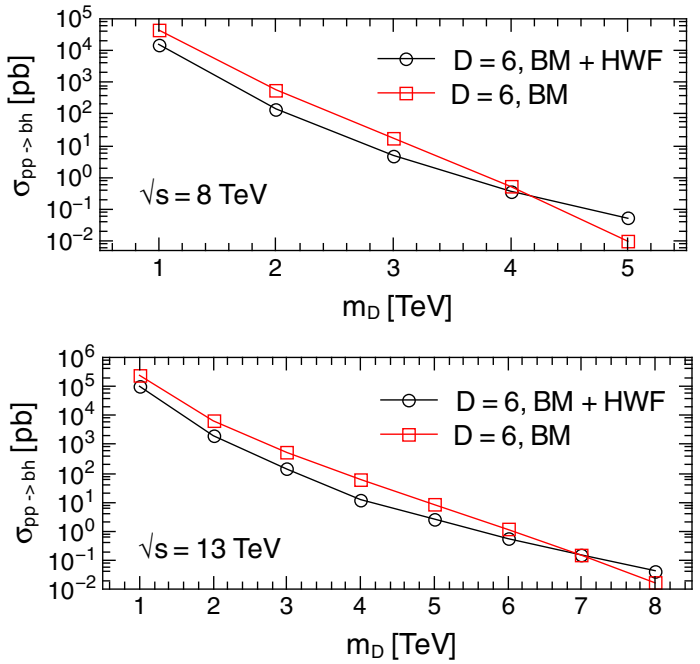

Fig. 5 Dependence of the total cross section $\sigma_{p p \rightarrow B H}$ on $m_{D}$ for $D=6$ and $\sqrt{s}=8 \mathrm{TeV}$ (top panel) or $\sqrt{s}=13 \mathrm{TeV}$ (bottom panel). Red squares represent the standard cross section, while black circles represent the HQM values

cross sections are very small. Figure 5 shows, for instance, that when $\sqrt{s}=8 \mathrm{TeV}$, the cross sections are of the order of $10^{4} \mathrm{pb}$ for $m_{D}=1 \mathrm{TeV}$, but they reduce to about $1 \mathrm{pb}$ for $m_{D}=4 \mathrm{TeV}$.

So far we mostly analysed cases with $m^{\min }=m_{D}$ in the standard scenario and compared with the HQM predictions for the same $m_{D}$. The tables in Appendix A present the dependence of the events ratio on $m^{\text {min }}$ and $m_{D}$, taken to be independent parameters for the standard scenario, for the same $m_{D}$ in the HQM case. The thick black lines in the tables show where the events ratio crosses over one: below the lines, the HQM predicts less BH events, whereas the standard simulations predict less such events above the lines. It is in particular interesting to compare the number of events predicted by the HQM with the number of events one expects to see in the standard case for the current lower bounds imposed by the LHC collaborations on $m_{D}$ and on the minimum mass that BHs can have [12-15,17]. In Fig. 6, we show a comparative analysis between the $\mathrm{BH}$ production cross sections (which ultimately translate into the number of events expected to be produced at the $\mathrm{LHC}$ ), and the distributions of $\mathrm{BH}$ masses for these two cases. In the plots, we assumed the strongest lower bounds on $m_{D}$ and $m^{\mathrm{min}}$ available [15,17], and as usual compared with the HQM predictions for the same $m_{D}$. It immediately appears that the HQM predicts more BH events. Upon examining Fig. 6 further, one also notices that the $\mathrm{BH}$ mass distributions differ: the HQM predicts that most $\mathrm{BHs}$ are produced with masses smaller than the values expected in the standard scenario. This is no surprise, given that we assumed the same value for $m_{D}$ in the two scenarios, the lower bounds imposed by the LHC groups on $m^{\text {min }}$ are stronger (higher values) than the bounds imposed on $m_{D}$, and that BHs with mass below $m_{D}$ are possible in the HQM. 
Fig. $6 \mathrm{BH}$ mass distributions and events ratio at the current LHC lower bounds for $m_{D}$ and $m^{\min }$
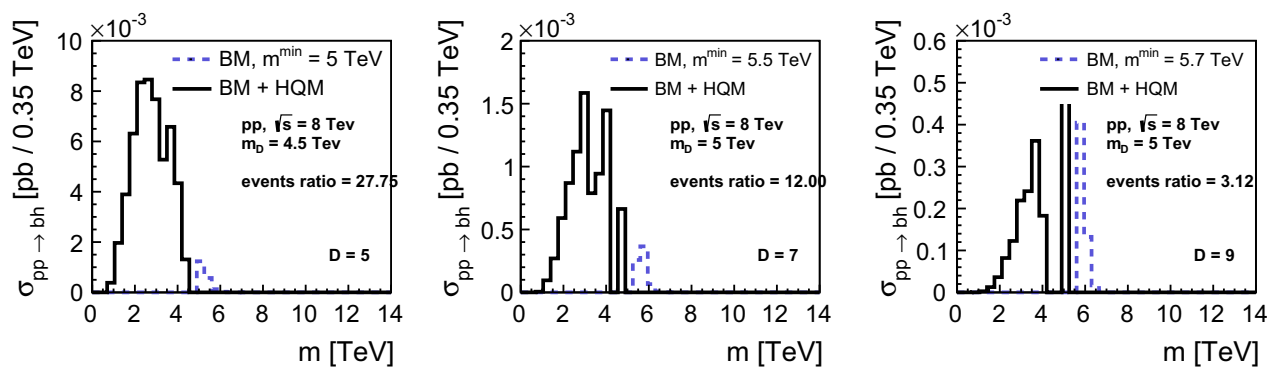

Fig. 7 HQM lower bound on $m_{D}$ obtained by requiring events ratio be approximately equal to one
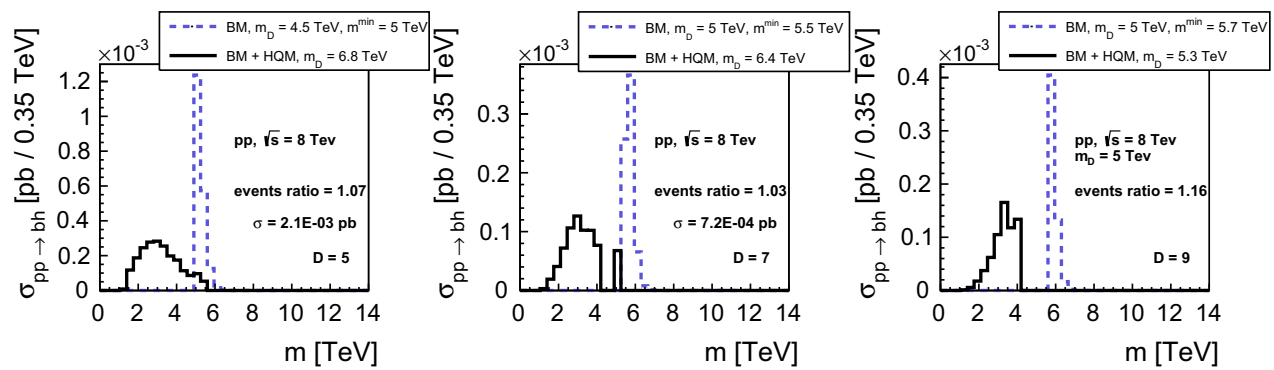

Fig. 8 HQM estimated BH mass distributions at $\sqrt{s}=13 \mathrm{TeV}$ with $m_{5}=6.8 \mathrm{TeV}, m_{7}=6.4 \mathrm{TeV}$ and $m_{9}=5.3 \mathrm{TeV}$
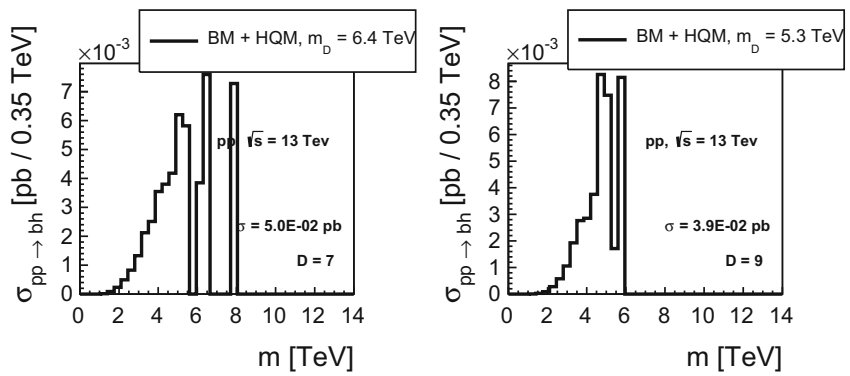

Since the HQM yields no minimum BH mass, the above comparison is not completely significant, and one should actually constrain only $m_{D}$ using experimental data in this scenario. We then determined the value of $m_{D}$ in the HQM formalism for which the number of $\mathrm{BH}$ events is expected to be the same as in the standard case at the current bounds. This means we set the BLACKMAX parameters for the standard case equal to the LHC bounds, and changed the value of $m_{D}$ in the HQM simulations. The results are presented in Fig. 7, from which one can see the events ratio is roughly equal to one for $m_{5} \simeq 6.8 \mathrm{TeV}, m_{7} \simeq 6.4 \mathrm{TeV}$, $m_{9} \simeq 5.3 \mathrm{TeV}$. In all cases, the HQM lower bounds on $m_{D}$ therefore appear stronger than in the standard case. The number of BH events expected at the LHC is about the same as in the corresponding standard scenario, but with a very different distribution of masses (most of the BHs are produced with masses lower than the minimum BH mass in the standard case). We thus caution the reader that in deriving these HQM lower bounds on $m_{D}$, we neglected the impact that the different HQM distributions of $\mathrm{BH}$ masses may have on the likelihood for the LHC collaborations to detect them. In fact, before the LHC collaborations reached the current limits, they also scanned the parameter space below those values.
With that disclaimer in mind, we can still consider the $\mathrm{HQM}$ bounds on $m_{D}$ and simulate the $\mathrm{BH}$ production at $\sqrt{s}=13 \mathrm{TeV}$. Figure 8 shows the expected distribution of $\mathrm{BH}$ masses in this case, with cross sections of the order of a few times $10^{-2} \mathrm{pb}$.

\section{Conclusions}

We investigated the implications of the HQM on the BH production cross sections at the LHC in the context of the ADD models with extra spatial dimensions. We used BLACKMAX to perform numerical simulations allowing us to compare both the production cross sections and the resulting $\mathrm{BH}$ mass distributions. The events ratio was used to express quantitatively the differences in the $\mathrm{BH}$ production cross sections in the two cases. We find that this ratio is always smaller for larger number of extra-dimensions, and that in each case it eventually increases with $m_{D}$ until it becomes larger than one. A wide range of cases are presented in Fig. 4 and the tables in Appendix A. When looking at the distribution of $\mathrm{BH}$ masses, in particular, we find that the HQM predicts most BHs are produced with masses smaller than the values expected in the standard scenario. 
We also compared the cross section predicted by the HQM with the standard one for the current lower bounds imposed by the LHC collaborations on the fundamental gravity scale $m_{D}$ and $m^{\min }$. We found that in the HQM case more BHs are produced. We thus determined new lower bounds on $m_{D}$, by finding the value of the fundamental gravity scale at which the number of $\mathrm{BH}$ events is expected to be the same as in the standard case at the current bounds: $m_{5} \simeq 6.8 \mathrm{TeV}, m_{7} \simeq 6.4 \mathrm{TeV}, m_{9} \simeq 5.3 \mathrm{TeV}$. Finally, we calculated the $\mathrm{BH}$ production cross sections for these new lower bounds at $\sqrt{s}=13 \mathrm{TeV}$. It will also be interesting to investigate the implications of the HQM for BH remnants at the LHC [10,30], or other modified decay channels [31], but we leave this analysis for future work.
Acknowledgments R.C. is partly supported by the INFN grant FLAG. N.A. and O.M. were supported by the grant LAPLAS 4 .

Open Access This article is distributed under the terms of the Creative Commons Attribution 4.0 International License (http://creativecomm ons.org/licenses/by/4.0/), which permits unrestricted use, distribution, and reproduction in any medium, provided you give appropriate credit to the original author(s) and the source, provide a link to the Creative Commons license, and indicate if changes were made.

Funded by SCOAP ${ }^{3}$.

\section{Appendix A: Events ratio}

See Figs. 9, 10, 11 and 12.
Fig. 9 Events ratio as a function of $m_{D}$ and $m^{\text {min }}$ for $D=5$ at $\sqrt{s}=8 \mathrm{TeV}$. The thick black line indicates the cross-over

Fig. 10 Events ratio as a function of $m_{D}$ and $m^{\text {min }}$ for $D=5$ at $\sqrt{s}=13 \mathrm{TeV}$. The thick black line indicates the cross-over events ratio, $\sqrt{\mathrm{s}}=8 \mathrm{TeV}, \mathrm{D}=5$

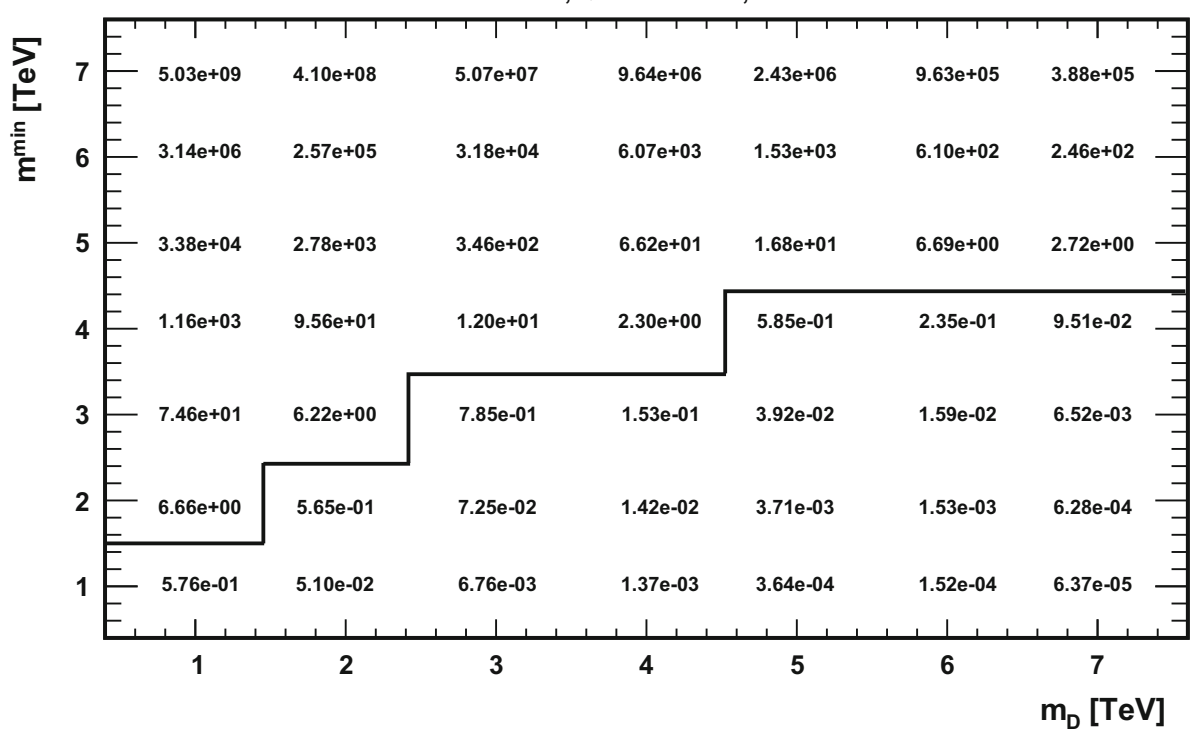

events ratio, $\sqrt{\mathrm{s}}=13 \mathrm{TeV}, \mathrm{D}=5$

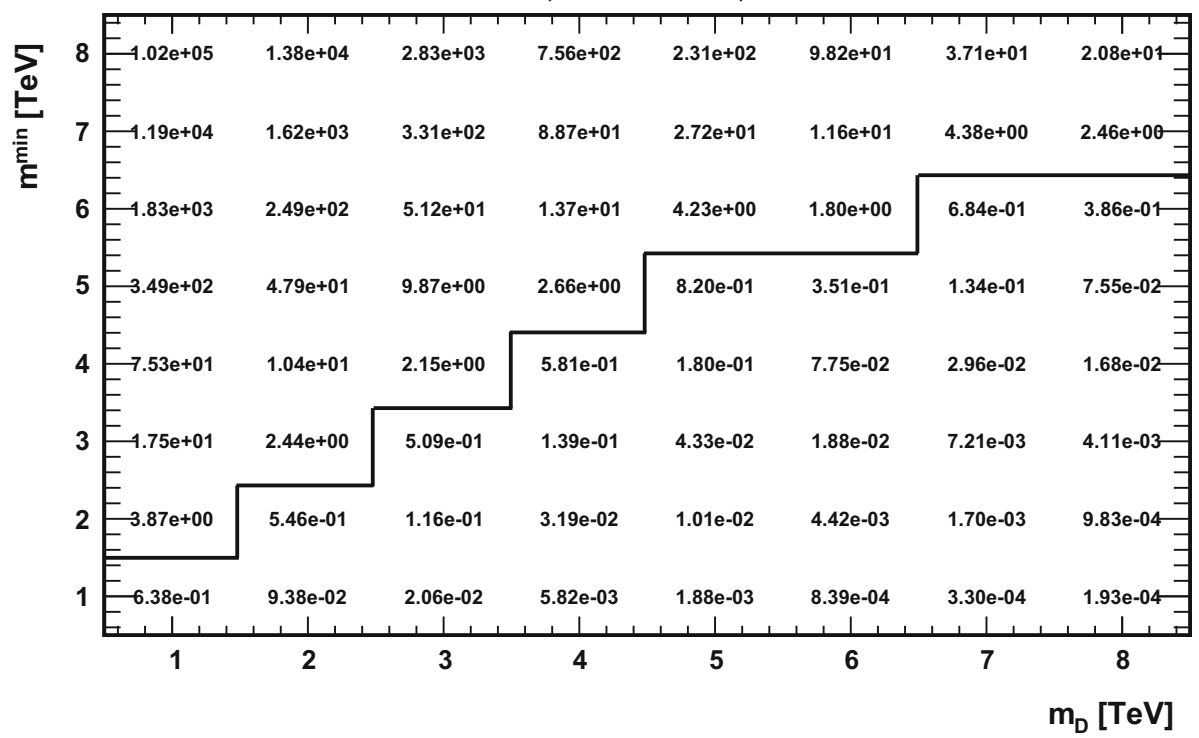


Fig. 11 Events ratio as a function of $m_{D}$ and $m^{\mathrm{min}}$ for $D=7$ at $\sqrt{s}=8 \mathrm{TeV}$. The thick black line indicates the cross-over

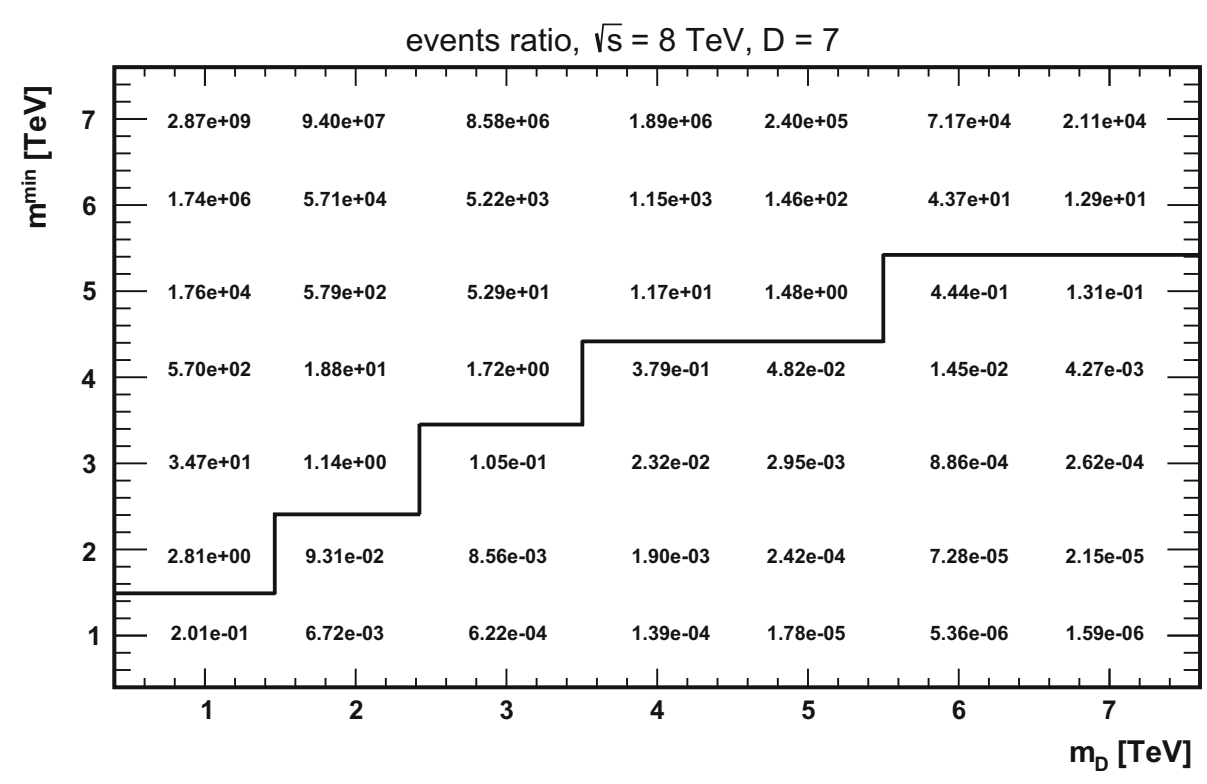

Fig. 12 Events ratio as a function of $m_{D}$ and $m^{\text {min }}$ for $D=7$ at $\sqrt{s}=13 \mathrm{TeV}$. The thick black line indicates the cross-over events ratio, $\sqrt{\mathrm{s}}=13 \mathrm{TeV}, \mathrm{D}=7$

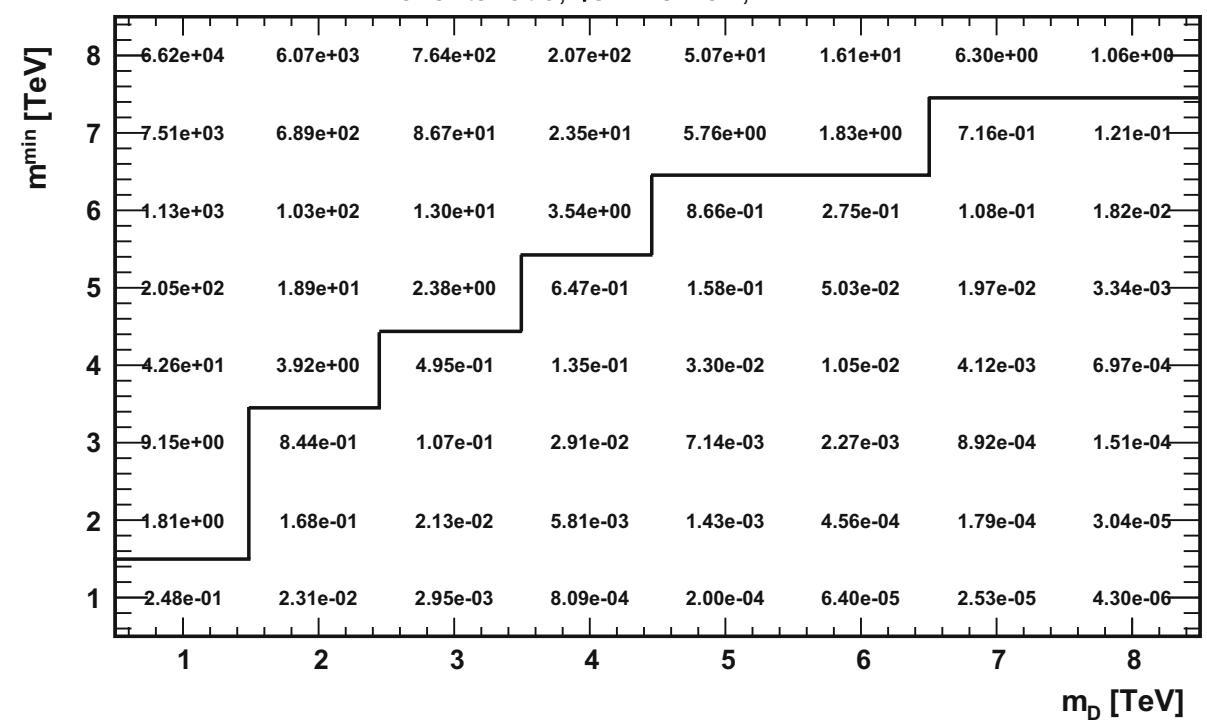

\section{References}

1. N. Arkani-Hamed, S. Dimopoulos, G.R. Dvali, Phys. Lett. B 429 , 263 (1998)

2. I. Antoniadis, N. Arkani-Hamed, S. Dimopoulos, G.R. Dvali, Phys. Lett. B 436, 257 (1998)

3. L. Randall, R. Sundrum, Phys. Rev. Lett. 83, 3370 (1999)

4. L. Randall, R. Sundrum, Phys. Rev. Lett. 83, 4690 (1999)

5. T. Banks, W. Fischler, A model for high-energy scattering in quantum gravity. arXiv:hep-th/9906038

6. R. Maartens, K. Koyama, Living Rev. Rel. 13, 5 (2010)

7. M. Cavaglia, Int. J. Mod. Phys. A 18, 1843 (2003)

8. S.B. Giddings, S.D. Thomas, Phys. Rev. D 65, 056010 (2002)

9. S. Dimopoulos, G.L. Landsberg, Phys. Rev. Lett. 87, 161602 (2001)

10. L. Bellagamba, R. Casadio, R. Di Sipio, V. Viventi, Eur. Phys. J. C 72, 1957 (2012)

11. G.L. Alberghi, L. Bellagamba, X. Calmet, R. Casadio, O. Micu, Eur. Phys. J. C 73, 2448 (2013)
12. G. Aad et al., JHEP 08, 103 (2014)

13. G. Aad et al., Phys. Rev. Lett. 112, 091804 (2014)

14. G. Aad et al., Phys. Rev. D 88, 072001 (2013)

15. S. Chatrchyan et al., JHEP 07, 178 (2013)

16. V. Khachatryan et al. [CMS Collaboration], Phys. Rev. D 91, 052009 (2015)

17. D.M. Gingrich, Int. J. Mod. Phys. A 30, 1530061 (2015)

18. R. Casadio, R.T. Cavalcanti, A. Giugno, J. Mureika, Horizon of quantum BHsin various dimensions. arXiv:1509.09317 [gr-qc]

19. R. Casadio, Localised particles and fuzzy horizons: a tool for probing quantum black holes. arXiv:1305.3195 [gr-qc]

20. R. Casadio, F. Scardigli, Eur. Phys. J. C 74, 2685 (2014)

21. R. Casadio, O. Micu, F. Scardigli, Phys. Lett. B 732, 105 (2014)

22. R. Casadio, O. Micu, D. Stojkovic, JHEP 1505, 096 (2015)

23. R. Casadio, O. Micu, D. Stojkovic, Phys. Lett. B 747, 68 (2015)

24. X. Calmet, R. Casadio, Eur. Phys. J. C 75, 445 (2015) 
25. R. Casadio, A. Giugno, O. Micu, Horizon quantum mechanics: a hitchhiker's guide to quantum black holes. arXiv:1512.04071 [hepth] (to appear in IJMPD)

26. D.-C. Dai, G. Starkman, D. Stojkovic, C. Issever, E. Rizvi, J. Tseng, Phys. Rev. D 77, 076007 (2008)

27. J.A. Frost, J.R. Gaunt, M.O.P. Sampaio, M. Casals, S.R. Dolan, M.A. Parker, B.R. Webber, JHEP 0910, 014 (2009)

28. D.M. Gingrich, Comput. Phys. Commun. 181, 1917 (2010)
29. M. Cavaglia, R. Godang, L. Cremaldi, D. Summers, Comput. Phys. Commun. 177, 506 (2007)

30. L. Alberghi, L. Bellagamba, X. Calmet, R. Casadio, O. Micu, Eur. Phys. J. C 73, 2448 (2013)

31. G.L. Alberghi, R. Casadio, A. Tronconi, J. Phys. G 34, 767 (2007) 